\title{
Transsaccadic Representation of Layout: What is the time course of Boundary Extension?
}

\author{
Christopher A. Dickinson and Helene Intraub \\ Department of Psychology, University of Delaware, Newark.
}

\begin{abstract}
How rapidly does boundary extension (BE) occur? Across experiments, trials included a 3-scene sequence ( $325 \mathrm{~ms} /$ picture), masked interval, and repetition of one scene. The repetition was the same view or differed (more close-up or more wide-angle). Observers rated the repetition as same, closer, or more wide-angle than the original view on a 5-point scale. Masked intervals were 100, 250, 625, or $1000 \mathrm{~ms}$ in Experiment 1, and 42, 100, or $250 \mathrm{~ms}$ in Experiments 2 and 3. BE occurred in all cases: identical views were rated as too "close-up", and distractor views elicited the rating asymmetry typical of BE (wider-angle distractors were rated as being more similar to the original than were closer-up distractors). Most important, BE was evident when only a 42-ms mask separated the original and test views. Experiments 1 and 3 included conditions eliciting a gaze shift prior to the rating test; this did not eliminate $\mathrm{BE}$. Results show that $\mathrm{BE}$ is available soon enough and is robust enough to play an on-line role in view integration, perhaps supporting incorporation of views within a larger spatial framework.
\end{abstract}

\section{Keywords}

Scene perception; Transsaccadic memory; Visuospatial memory; Boundary extension; Spatial cognition

\section{Transsaccadic Representation of Layout: What is the time course of Boundary Extension?}

We can never see the surrounding visual world all at once. Instead, we must sample it a part at a time through successive movements of the eyes and head. An interesting aspect of memory for a single view of a scene is that it will often be remembered as having shown more of the scene than was available in the sensory information-observers remember seeing beyond the edges of the view. This is referred to as boundary extension (BE; Intraub \& Richardson, 1989). Although BE is an error with respect to the stimulus, it provides a good prediction of the world beyond the view. For this reason, it has been suggested that BE might serve an adaptive function in scene representation by placing each view within its larger spatial framework (Intraub, 2002, 2007; Intraub, Bender, \& Mangels, 1992).

This hypothesis has received support from both behavioral and neuroimaging data. Behavioral research has shown that BE is not present in memory for all pictures of objects (e.g., an object on a blank background), but only for those in which the background depicts part of the visual world (i.e., scene layout; Gottesman \& Intraub, 2002, 2003; Intraub, Gottesman \& Bills, 1998). A similar distinction between pictures that include scene layout and those that do not

Correspondence concerning this article should be addressed to Christopher A. Dickinson or Helene Intraub, University of Delaware, Newark, Delaware, 19716. cdickins@udel.edu or intraub@udel.edu.. 
is reflected in the heightened neural responses of the parahippocampal place area (PPA) to pictures of scene layout (Epstein \& Kanwisher, 1998). More recently, an fMRI study of brain activation in the presence of BE revealed that indeed PPA was highly activated when BE occurred (Park, Intraub, Yi, Widders, \& Chun, 2007). These experiments indicate that BE is part of the representation of scene layout. The purpose of the present research is to determine the early time course of BE. Specifically, at what stage of processing does BE occur?

In the typical experiment, short series of photographs were presented for multi-second durations (e.g., $15 \mathrm{~s} \mathrm{each}$ ), and memory was tested minutes to 48 hours later (e.g., Candel, Merckelbach, \& Zandbergen 2003; Gottesman \& Intraub, 2002; Intraub et al., 1992, 1998; Intraub \& Richardson, 1989; Mathews \& Mackintosh, 2004). These results suggest that BE may be a long-term phenomenon. In fact, Koriat, Goldsmith, and Pansky (2000), in their review of memory errors, grouped BE with memory errors for text in which "... schematic knowledge is used to make inferences and suppositions that go beyond the actual input event, ... (p. 494)". They noted that schema-induced errors such as these tend to increase over time as memory becomes less detailed. If $\mathrm{BE}$ requires long retention times to occur, then it could not play a role on-line during visual scanning. It might be the case that at the earliest stages of processing (e.g., between fixations), visual memory for a just-fixated view might be strong enough to maintain fairly veridical boundaries.

However, an experiment described in Previc and Intraub (1997) demonstrated, somewhat surprisingly, that BE did occur rapidly enough to be observed across a series of perception/ action cycles during drawing. Observers viewed four photographs for $15 \mathrm{~s}$ each and then drew them from memory. Another group drew them from a projected image on a screen at the front of the room. As expected, BE occurred in the memory group. What was striking was that BE also occurred in drawings made by observers who could see the photographs. Does this mean that $\mathrm{BE}$ occurs during perception?

To answer this question it is important to consider the task carefully. While an observer is literally looking at a picture, he or she can see where the picture ends, and can imagine what would likely exist in the world beyond the edges of the view. Both the sensory information and the expected layout beyond the edges are part of the observer's representation. Unlike wellknown perceptual illusions (e.g., the Mueller-Lyer illusion), BE does not occur while the stimulus is in view. However, when drawing, observers did not maintain fixation on the projected image. They shifted their gaze from the projected image to the paper on their desks, thus relying on memory of the view while they drew. They occasionally would look up and sample the image then look down and draw again, alternating between sensory perception and memory. This suggests that BE occurred at least within seconds after the sensory image was gone, while the observer was drawing.

Consistent with this observation, Intraub, Gottesman, Willey, and Zuk (1996) demonstrated that BE occurs for photographs presented in brief rapid serial visual presentation (RSVP) sequences when memory was tested for the last item in the sequence only 1 second later (see also Bertamini, Jones, Spooner, \& Hecht, 2005). Given these results, two possibilities are clear. First, BE might occur a second or more following offset of the stimulus, suggesting it is a very short-term memory error-not rapid enough to play a role in transsaccadic memory, but available soon after perception. Alternately, BE might occur as soon as the sensory input is gone. Rather than occurring "in memory," instead it might be part of the unfolding process of scene perception, which involves a rapidly changing cycle of sensory perception and memory.

If $\mathrm{BE}$ is available during the time between saccades (i.e., transsaccadic memory; see Irwin, 1991, 1993), then we would expect to see it under the following three conditions. First, it should occur following a brief glimpse of a scene (analogous to the duration of a single fixation). BE 
is known to occur following presentations as brief as $250 \mathrm{~ms}$ and $333 \mathrm{~ms}$ (Bertamini et al., 2005; Intraub et al., 1996; 500 ms: Intraub, Hoffman, Wetherhold, \& Stoehs, 2006).

Second, it should be evident following a gap in sensory input commensurate with a "typical" saccade (on the order of 30 to $50 \mathrm{~ms}$; Rayner, 1998). However, retention times briefer than 1 $s$ have not been tested. Third, BE would need to survive a gaze shift caused by a change in the position of the eyes or the head. Recent research shows that BE occurs following a single eye movement when memory is tested $2 \mathrm{~s}$ later (Intraub et al., 2006), but it is not known whether it occurs when tested immediately after a gaze shift. It is possible that the process of planning and executing a gaze shift might delay the onset of $\mathrm{BE}$ for a couple of seconds, thus preventing its inclusion in transsaccadic memory.

\section{Might Early Memory Buffers Prevent BE?}

It may be that early visual buffers maintain a fairly veridical representation of layout, essentially protecting the representation from distortions. Iconic memory has been categorized as a highcapacity, brief-duration, veridical buffer that is disrupted by masking by both luminance and pattern. So, for example, if a single fixation on a scene were followed by a fixation on an empty region of space, then a veridical representation of the spatial expanse of that view might be maintained in an iconic representation (i.e., as informational persistence) for 100 to $300 \mathrm{~ms}$ (Irwin \& Brown, 1987; Irwin \& Yeomans, 1986).

What if, however, a single fixation on a view of a scene is followed by another fixation whose contents would disrupt an iconic representation, or alternately, an iconic representation isn't maintained across a gaze shift? Visual short-term memory (VSTM) has been categorized as a longer lasting (i.e., multi-second) buffer that does not maintain a literal copy of the physical visual stimulus (i.e., it is not a point-by-point copy) and that is capacity-limited but not disrupted by visual masking (Irwin, 1991; Phillips, 1974). Numerous studies suggest that VSTM representations are not literal copies of the display (Gordon \& Irwin, 1996; Henderson, 1994; Hollingworth, Hyun, \& Zhang, 2005; Irwin, 1991, 1992; Irwin \& Andrews, 1996; Olson \& Jiang, 2004; Phillips, 1974).This does not imply, however, that VSTM representations contain no visual information. Here we ask whether VSTM might maintain a veridical representation for a brief interval following a picture's offset, thus preventing BE.

\section{Might Gaze Shifts Delay BE?}

If $\mathrm{BE}$ occurs rapidly enough to support view integration on-line, it would not be of use unless it could also survive a shift in gaze. In prior boundary extension research, stimulus and test views have always been presented in the same physical location. Might the demands of attention/motor systems engaged during gaze shifts early in processing either delay the onset of BE or cause memory for boundaries simply to be poor-causing errors but not the strong unidirectional error that characterizes BE? Numerous studies have demonstrated that gaze shifts suppress a variety of cognitive processes, whereas others appear to continue across them unimpeded (for a review, see Irwin \& Brockmole, 2004). A brief examination of the known properties of transsaccadic memory might shed some light on whether BE might be included in transsaccadic memory.

Most experiments on transsaccadic memory have focused not on scenes as a whole, but on the properties of individual objects that are remembered across a saccade. In general, results suggest that representations of structural descriptions of objects are retained (CarlsonRadvansky \& Irwin, 1995; Carlson-Radvansky, 1999; Verfaillie \& De Graef, 2000; Verfaillie, De Troy, \& Van Rensbergen, 1994), but there is also evidence for retention of specific visual information (object orientation: Henderson \& Siefert, 1999, 2001; but not detailed object contours: Henderson, 1997). 
Boundary extension, however, involves an extrapolation of layout. Although the literature on transsaccadic memory has not focused on scene layout, there have been several studies that have focused on retention of spatial relations. These provide evidence that spatial information is available in transsaccadic memory. It has been shown that information that specifies the structural relations of parts of a single object is included in a transsaccadic representation (Carlson-Radvansky \& Irwin, 1995). In addition, information about the configuration, or the spatial relations among different objects, can also be represented (Carlson-Radvansky, 1999; Deubel, 2004; Germeys, de Graef, Panis, van Eccelpoel, \& Verfaillie, 2004). The question we ask is whether this transsaccadic representation includes extrapolated layout (BE) or instead maintains a more veridical representation of the view.

The Current Experiments-To explore the early time course of BE and test its resiliency to shifts in gaze, in all three experiments we used Intraub et al.'s (1996) three-picture RSVP method. Three pictures were presented for $325 \mathrm{~ms}$ each in a continuous sequence followed by a masked retention interval and subsequent boundary memory test. There were three reasons for choosing this method. First, by embedding a target picture in a rapidly changing series, we could approximate the dynamic nature of visual scanning. ${ }^{1}$ Second, the design allowed us to test boundary memory following presentations of 0,1 , or 2 intervening items, thus allowing us to determine if factors such as conceptual masking (Intraub 1984; Potter, 1976) during successive presentation might influence BE. Third, the rapidity of input coupled with observer uncertainly about which picture would be tested would minimize the observer's ability to develop verbal strategies (e.g., "the man's head is .5 cm from the top") for remembering boundary placement over the course of the session.

In all three experiments, on each trial, the observer was required to rate the repeated scene as being the same view or a more close-up view or a more wide-angle view than before on a 5point scale (Intraub \& Richardson, 1989). Across experiments the interval between offset of the last picture and onset of the test picture was always masked and it ranged between $42 \mathrm{~ms}$ (comparable to the duration of a saccade) and $1 \mathrm{~s}$ (to replicate earlier research). To test the effect of a shift in gaze on boundary extension, in Experiments 1, 3a, and 3b, test pictures were presented either in the same location as the RSVP sequence, or to the left or right side of the screen.

Depending on the experiment, when a scene repeated, it could be the same, a more close-up view, or a more wide-angle view than one of the pictures in the presentation sequence. In this way, all the patterns of response that are diagnostic of boundary extension could be addressed following each masked interval. These patterns have been replicated in many studies (e.g., Bertamini et al., 2005; Gottesman \& Intraub, 2002; Intraub et al., 1992, 1998; Intraub \& Richardson, 1989). The three patterns of interest are as follows:

1. When the target picture and the test picture are identical close-ups, observers tend to reject the test picture as being the same, reporting instead that it is more close-up than the target picture. This was tested in Experiments 1-3.

2. Target pictures that are tight close-ups yield more boundary extension than wider angle views of the same scene; in fact, wider angle views tend to yield no directional distortion (see Gottesman \& Intraub, 2002; Intraub et al., 1992; Intraub \& Berkowits, 1996). This was tested in Experiment 2.

\footnotetext{
${ }^{1}$ Of course, there are several aspects of visual scanning that RSVP does not simulate: for example, the peripheral information made available by the spatial attention shift preceding an eye movement (Deubel \& Schneider, 1996; Hoffman \& Subramaniam, 1995; Irwin \& Zelinsky, 2002; Kowler, Anderson, Dosher, \& Blaser, 1995), or the brief period of saccadic suppression that occurs between fixations (see Volkmann, 1986). However, it does allow us to approximate the changes in visual input that result from gaze shifts, as well as the temporal pacing of these changes.
} 
3. When a close-up is the target, and a wider view is presented at test, observers rate the pair as being more similar than when the reverse is the case. This is because $\mathrm{BE}$ for a closer target causes it to be remembered as looking more like the wider angle test picture. This asymmetry was also observed in neural responses to dissimilar pictures in scene selective brain regions (Park et al., 2007). This signature pattern was tested in Experiment 2.

\section{Experiment 1}

In Experiment $1 \mathrm{BE}$ was tested for a single picture immediately following a three-picture RSVP sequence and a masked interval of $1 \mathrm{~s}, 625 \mathrm{~ms}, 250 \mathrm{~ms}$, or $100 \mathrm{~ms}$. We selected the latter three intervals as coarse divisions of the 1-s interval after which BE is known to occur (Bertamini et al., 2005; Intraub et al., 1996). To provide the most sensitive test of BE on half the trials the same close-up served as both target and test picture. Thus in the critical case for testing time course (when the target picture is in the final serial position of the RSVP sequence), the same close-up view of a complex scene was interrupted by a 100-ms masked interval. To ensure that observers were focused on the task and were using the scale appropriately, on the remaining trials, scenes were divided into two sets: in one a close-up target was tested with a more wideangle view of the same scene, and in the other, a wider angle view was tested with a close-up version.

Finally, to determine if BE survives a gaze shift between the target picture and the test picture, the test picture appeared equally often in the same location as the target (center screen) or shifted to the right or left side of the screen. If BE does not occur at intervals briefer than $1 \mathrm{~s}$, or if a gaze shift disrupts the extrapolation of expected surrounding space, then BE cannot play an on-line role in view integration during active scanning.

\section{Method}

Participants-A total of 144 University of Delaware undergraduates, fulfilling a requirement for an introductory psychology course, participated in the experiment. All reported having normal or corrected-to-normal vision and normal color vision.

Apparatus-All stimuli were presented on a 21" flat-screen CRT monitor in 32-bit color at a resolution of $1024 \times 768$ pixels and a refresh rate of $120 \mathrm{~Hz}$ that was driven by a video card with $128 \mathrm{mb}$ of video memory. Stimulus presentation was controlled by a Pentium-based PC running Microsoft Windows XP. The software was based on a template program supplied by SR Research Inc. written in C that used Simple DirectMedia (SDL) v.1.2.9 to access the video hardware. The viewing distance was approximately $80 \mathrm{~cm}$, and on average, pictures subtended $9.2^{\circ} \times 10.2^{\circ}$ of visual angle (widths ranged from $5.9^{\circ}$ to $13.7^{\circ}$; heights ranged from $8.9^{\circ}$ to $\left.10.4^{\circ}\right)$.

Stimuli-Stimuli were 96 color photographs that depicted people engaging in various activities; for example, a football player kicking a football, a man tossing a pizza, and a couple dancing. Some of the images were copied, with permission, from The Big Box of Art database (Hemera Technologies, Inc.); others were downloaded from the Internet. All stimuli were presented on a gray background. Of the 96 pictures, 32 served as targets (i.e., to-be-tested pictures: 2 in the practice trials and 30 in the experimental trials). The other 64 pictures served as fillers in the presentation sequence (i.e., the two non-target pictures in the RSVP triad). A given scene (close or wide version) was always presented with the same filler items. Each target scene could be presented in its close-up version or its more wide-angle version, as is illustrated in Figure $12^{2}$ Close-ups were created by enlarging the wide-angle version by $8 \%$ to $21 \%$ in area and then cropping the enlarged version to be the same size as the original. Thus, 
both versions were the same size, but the wide-angle version showed a larger amount of background surrounding the main object or objects.

Design and Procedure-A depiction of a trial sequence is shown in Figure 2. Each selfinitiated trial began with a central fixation point that remained on screen for $500 \mathrm{~ms}$. The RSVP sequence followed ( $325 \mathrm{~ms} /$ picture) in the center of the screen. The target picture appeared equally often in serial position 1, 2, or 3. Observers did not know which picture would ultimately be tested. The RSVP sequence was immediately followed by a masked interval of $100,250,625$, or $1000 \mathrm{~ms}$ (between groups). For a target picture that appeared in serial position 3 , the retention interval would be the same as the duration of the masked interval; for target pictures presented earlier in the sequence, the retention interval would be equal to the duration of the masked interval plus the duration of the picture or pictures that followed it.

The mask was a pattern mask that filled the screen and had a dynamically changing central portion. As is shown in Figure 2, the central component was a schematic face that subtended $5.5^{\circ} \times 5.5^{\circ}$ of visual angle. A sequence of 4 different faces was shown, and each face was visible for either $150 \mathrm{~ms}$ or was terminated when the masked interval ended. The repeated onsets were intended to minimize implicit verbalization and keep the observers' eyes on the center of the screen before the test picture appeared. At the end of the masked interval, the test picture was shown either in the display's center (referred to as maintain-fixation trials) or to the left or right of center (referred to as shift-gaze trials), shifted by an average of $5.8^{\circ}$ (ranging from $4.2^{\circ}$ to $7.4^{\circ}$ ). It appeared equally often at each location, without warning. On trials in which the test picture appeared in a different location than the RSVP sequence, the interval between the offset of the final picture in the RSVP sequence and the beginning of an observer's first fixation on the test picture would be defined by the time required for the observer to shift his or her gaze to the test picture following its onset. Otherwise, this interval would be equal to the masked interval.

Observers were asked to rate whether the test picture showed the same view, a more close-up view, or a more wide-angle view than before using a 5-point Likert scale. The alternatives (and their corresponding numerical values) were "much closer up (-2)," "a little closer up (-1)," "the same (0)," "a little farther away (1)," and "much farther away (2)." The test picture was visible until the observer clicked one of these choices with the mouse. Observers then indicated how confident they were about their response by clicking "sure (3)," "pretty sure (2)," "not sure (1)," or "don't remember that picture (0)." The fixation point for the next trial then appeared and the observer initiated the trial by clicking the mouse.

There were a total of 30 trials. On 15 trials the target and test picture were identical close-ups (CC trials). On 14 trials the target and test picture were different views of the same scene; half the time a close-up was the target and the test picture was a more wide-angle view $(C W$

trials) and half the time the reverse was true (WC trials). Each target picture was tested in only one of these conditions across observers. The $30^{\text {th }}$ trial was always a "dummy" trial in which the target picture was tested with the same view. This trial had to be added to allow us to show each target at each serial position equally often across observers, while at the same time having an equal number of $\mathrm{CW}$ and WC trials. Responses made on this trial were not included in the analyses.

\footnotetext{
${ }^{2}$ All pictures that were used as targets in Experiment 1 were downloaded from the Internet. To protect against copyright infringement, we show an example used in Experiments 2 and 3 only.
} 


\section{Results and Discussion}

Observers were rather confident of their ratings; on average, $21 \%, 57 \%$, and $20 \%$ of their responses were rated as "sure," "pretty sure," and "not sure", respectively. They reporting not recognizing the test picture on only $2 \%$ of the trials, and these were excluded from analysis. A $4 \times 2$ (masked interval $\times$ side of display) mixed-design ANOVA comparing observers' mean boundary ratings for test pictures presented on the left vs. right side of the screen revealed no main effect of location, $F(1,140)=1.73$, n.s., and no interaction with the masked interval, $F$ $<1$. Observers' mean boundary ratings in these two conditions were subsequently collapsed across this factor.

Critical CC trials: Targets and test pictures are identical close-ups-Figure 3 (left) shows the mean boundary rating at each serial position (collapsed over the spatial position of the test picture). The $95 \%$ confidence intervals revealed that BE occurred at each serial position for each masked interval. Thus boundary extension occurred even at the briefest interval tested - when the final picture in a sequence was repeated only $100 \mathrm{~ms}$ later. To determine if the size of the BE effect was influenced by the duration of the masked interval, a $3 \times 4$ (serial position $\times$ retention interval) mixed-design ANOVA was conducted. It revealed no main effect of the masked interval's duration, $F(3,140)=1.16$, n.s., no effect of serial position, $F<1$, and no interaction, $F<1$. The lack of a serial position effect shows that the onset of new meaningful pictures during RSVP did not disrupt incorporation of the extrapolated region into the spatial representation of the scenes - that is, there was no effect of conceptual masking (Intraub, 1984;Potter, 1976).

Figure 3 (right panel) shows the mean boundary rating as a function of the spatial location of the test picture for each masked interval (collapsed over serial position). As shown by the $95 \%$ confidence intervals in the figure, boundary extension occurred whether or not a shift in gaze intervened between presentation and test. Observers were never forewarned about the location of the test picture, yet when it shifted away from center screen, the concomitant gaze shift had no inhibitory effect on BE. The expanded representation of layout clearly survived the shift in attention and subsequent gaze shift, suggesting that $\mathrm{BE}$ is available during the time course of transsaccadic memory.

CW and WC Trials-The mean boundary ratings for CW trials and for WC trials (collapsed over serial position and spatial position) for each masked interval are shown in Figure 4 (left and right panels, respectively). Observers were able to recognize the presence of distractors, and were clearly using the scale appropriately. Consistent with the occurrence of BE, the right panel shows that observers were quite good at recognizing when the test picture was more close-up than the target, whereas the left panel shows that more wide-angle test pictures were sometimes mistaken as being the same as the target.

\section{Experiment 2}

Experiment 1 showed that a $100 \mathrm{~ms}$ interruption was sufficient to elicit BE. In Experiment 2 we decreased the briefest interval further to $42 \mathrm{~ms}$ (commensurate with a saccade); intervals tested were $250 \mathrm{~ms}, 100 \mathrm{~ms}$, and $42 \mathrm{~ms}$. To enhance the observer's ability to retain a veridical representation, the RSVP sequence and test picture were always in the same location (center screen). In addition, we sought to obtain converging evidence for BE through implementation of a design used in many prior BE studies (e.g., Intraub et al., 1998; Intraub \& Richardson, 1989), in which targets were either close-up or wide-angle views of a scene, and the test picture was either the same view as the target or its complement. This yielded four different test conditions: 1) Close-up view tested with the same close-up view (CC trials), 2) Wide-angle view tested with the same wide-angle view (WW trials), 3) Close-up view tested with the wideangle version of the scene (CW), and 4) Wide-angle view tested with the close-up version of 
the scene (WC). Scenes were counterbalanced across these four conditions. In this way we could determine if all three patterns that are diagnostic of boundary extension (i.e., boundary extension for CC trials, little or none for WW trials, and a CW-WC asymmetry, as discussed in the introduction) would occur.

\section{Method}

Participants-A total of 108 University of Delaware undergraduate students, fulfilling a research-participation requirement for an introductory psychology course, participated in the experiment. All reported having normal or corrected-to-normal vision and normal color vision, and none participated in the previous experiment.

Apparatus and Stimuli-The apparatus and stimuli were the same as in the previous experiment with the exception of the following differences. In the current experiment there were a total of 114 pictures of real-world scenes used, with 38 serving as targets and the remaining 76 serving as fillers for the presentation sequences. Here we added 6 new sequences to the 32 sequences used in the previous experiment.

Procedure and Design-The procedure was the same as in Experiment 1 with the exception that all stimuli and test pictures were presented in the center of the screen.

There were a total of 36 trials. On half of the trials, the target was a close-up view; on the other half, it was a wide-angle view. Within each of these conditions, on half of the trials the test picture was the same view as the target picture (CC, WW); on the other half, it was a different (i.e., the other) view (CW, WC). This yielded 9 trials for each target view-test view combination. Within each of these four target-test combinations, the target picture appeared in serial position 1,2 , and 3 of the initial presentation equally often, yielding three trials per cell of the design. Across observers, every target picture appeared in each of these conditions equally often. Each observer was tested with only one of the three masked intervals $(42,100$, and $250 \mathrm{~ms}$ ), with 36 observers being tested with each masked interval.

\section{Results and Discussion}

As in Experiment 1, observers were rather confident, rating their responses as "sure," "pretty sure," and "not sure" on $27.5 \%, 53 \%$, and $17 \%$, of the trials respectively. They failed to recognize test pictures on only $2.5 \%$ of the trials, and these were excluded from all analyses.

BE ratings for the final picture in each sequence-To examine the early time course of BE, here we focus on those trials on which the target picture appeared in serial position 3. All three patterns consistent with BE were obtained. First, as in Experiment 1, CC trials yielded boundary extension at all masked intervals-even the 42-ms masked interval. Figure 5 shows the mean boundary rating and $95 \%$ confidence interval for each serial position at each masked interval. The upper left panel shows that CC trials yielded boundary extension at each masked interval. Thus, the results show that on average, a 42-ms interruption in the sensory input was sufficient to prevent observers from recognizing the repetition of a close-up view-instead, they remembered the first presentation as having extended boundaries.

Second, wide-angle views yielded less BE than their close-up counterparts. The mean boundary ratings for WW trials for each masked interval are shown in Figure 5 (upper right). A $3 \times 2$ (masked interval $\times$ test type) mixed-design ANOVA on observers' mean boundary ratings revealed that CC trials yielded more BE than WW trials, $F(1,105)=12.36, p<.05$, partial $\eta^{2}=.11$, and the lack of interaction with masked interval duration $(F<1)$ indicates that this same pattern occurred for each masked interval. 
Third, a robust asymmetry in responses to the $\mathrm{CW}$ and $\mathrm{WC}$ trials, as is shown in Figure 5 (lower left and lower right), provided strong additional support that BE was occurring at these intervals. The same pair of pictures was rated differently (with respect to how different they were from one another) depending on their order (close-up first or wide-angle first). A $3 \times 2$ (masked interval $\times$ test type) mixed ANOVA on the absolute value of the mean boundary scores on $\mathrm{CW}$ and WC trials revealed a main effect of test type, $F(1,105)=40.81, p<.05$, partial $\eta^{2}=.28$, indicating that $\mathrm{CW}$ test pictures were rated as being closer to "same" than were WC test pictures. The lack of an interaction with masked interval duration $[F(2,105)=1.57$, n.s. $]$ again indicates that this same pattern occurred for each masked interval. Taken together, these patterns suggest that $\mathrm{BE}$ is part of the mental representation of a scene very rapidly after stimulus offset. They also suggest that BE occurs rapidly enough to be available from one fixation to the next.

We also found that more BE occurred for the longest masked interval than for the shortest one. A one-way ANOVA on mean boundary ratings for $\mathrm{CC}$ trials revealed a main effect of masked interval duration, $F(2,105)=3.31, p<.05$, partial $\eta^{2}=.06$, and planned comparisons revealed that mean ratings for the 250 -ms interval were larger than mean ratings for the 42 -ms interval $(-.47$ vs. -.19$), t(70)=2.69, p<.05$. This small difference could have been the result of a fading visual representation that was attenuating $\mathrm{BE}$ at the shortest interval. The results of a recent experiment, however, suggest that this difference may be related to the memory load resulting from the 3-picture stimulus sequences (Intraub \& Dickinson, 2006). When we presented observers with only a single stimulus for $250 \mathrm{~ms}$ and then tested their memories for the views (using the same procedure as in the current experiment), we found no difference in the amount of BE that occurred following $42 \mathrm{~ms}$ and 250 ms masked intervals.

BE ratings for pictures in serial positions 1 and 2-Analysis of responses to pictures in serial positions 1 and 2 showed that BE was not eliminated by the onset of new pictures (i.e., conceptual masking; Potter, 1976). The same three critical data patterns diagnostic of BE occurred on these trials. First, as is shown in the upper left panel of Figure 5, CC trials yielded $\mathrm{BE}$ at all masked intervals. Second, a $3 \times 2 \times 2$ (masked interval $\times$ test type $\times$ serial position) mixed ANOVA comparing mean boundary ratings on CC trials and on WW trials revealed that less BE occurred for WW trials than for CC trials, $F(1,105)=27.39, p<.05$, partial $\eta^{2}=$. 21 . There was no significant interaction of test type with either masked interval, $F(2,105)=$ 1.03 , n.s., or serial position, $F(1,105)=2.80$, n.s., indicating that this pattern occurred at each serial position and each masked interval. Third, a $3 \times 2 \times 2$ (masked interval $\times$ test type $\times$ serial position) mixed ANOVA on the absolute value of the mean boundary scores on CW and WC trials revealed a main effect of test type, $F(1,105)=59.74, p<.05$, partial $\eta^{2}=.36$. Further, this main effect of test type did not interact with either masked interval, $F<1$, or with serial position, $F<1$, indicating that this asymmetry occurred at each serial position and each masked interval.

\section{Experiments $\mathbf{3 a}$ and $\mathbf{3 b}$}

Experiment 2 demonstrated that BE occurs rapidly enough to play a role in view integration during visual scanning. In Experiments $3 \mathrm{a}$ and $3 \mathrm{~b}$, we therefore returned to the question of whether or not a shift in gaze would influence BE. In Experiment 3a, as in Experiment 2, the masked intervals tested were $250 \mathrm{~ms}, 100 \mathrm{~ms}$, and $42 \mathrm{~ms}$. Test pictures were presented in either the same spatial location as the RSVP sequence or in a different (non-overlapping) location. In Experiment 3b, we recorded observers' eye movements to determine the extent to which they were maintaining central fixation during stimulus presentation and to determine the duration of the saccade-defined interval between the end of the stimulus sequence and the beginning of observers' first fixations on the test items in shift-gaze trials. 
All trials were CC trials (close-ups tested by close-ups). Unlike Experiment 1, position of the test picture was blocked so observers always knew where the test picture would appear. We reasoned that by eliminating this uncertainty we would maximize our ability to detect any subtle influences of a gaze shift on memory for the expanse of a view. To determine if the time between the offset of the last picture and the beginning of the saccade would influence BE, we included the same 3 masked intervals as in Experiment 3a. The end of the interval signaled the start of a saccade.

\section{Method}

Participants-In Experiment 3a, 108 University of Delaware undergraduate students, fulfilling a research-participation requirement for an introductory psychology course, participated. In Experiment 3b, 36 individuals (5 college graduates who were paid \$8/hour, and 31 students) participated. All reported having normal or corrected-to-normal vision and normal color vision, and none participated in either of the previous experiments.

Apparatus and Stimuli-The apparatus was the same as in the previous experiment. The stimuli included only the close-up versions of targets and test pictures from the previous experiment. In Experiment 3b, we used an EyeLink II video-based eye tracking system to collect eye movement data (SR Research). Eye position was sampled at $500 \mathrm{~Hz}$, the system's spatial resolution was estimated to be less than $0.4^{\circ}$, and head position and viewing distance were fixed with a chinrest.

Design and Procedure-There were a total of 36 trials. Stimuli and test pictures were shown on either the left side of the screen or the right side-no items were presented in the screen's center, and there was no spatial overlap between the stimulus sequence and the test picture when they appeared on different sides of the screen. When the two were presented in different locations, the average center-to-center distance was $11.8^{\circ}$ (ranging from $8.4^{\circ}$ to $14.2^{\circ}$ ). On half of the trials, the test picture was shown on the same side of the screen as the stimulus sequence (maintain-fixation trials); on the other half, it was presented on the other side of the screen (shift-gaze trials). Within each of these conditions, on half of the trials the stimulus sequence was presented on the left side of the screen; on the other half, it was presented on the right side. This yielded 9 trials for each stimulus side-test side combination. Otherwise, the procedure was the same as in the previous experiment.

These conditions were blocked, and observers were informed of the stimulus and test locations at the start of each block. The sequence of blocks was counterbalanced across observers. Within each of these four stimulus-test combinations (test was on the same side: LL, RR; test was on the other side: LR, RL), the target picture appeared in serial position 1,2, and 3 of the initial presentation equally often, yielding three trials per cell of the design. Across observers, every picture appeared in each of these conditions equally often. Each observer in Experiment 3a was tested with only one of the three masked intervals $(42,100$, and $250 \mathrm{~ms})$, with 36 observers being tested with each masked interval. In Experiment 3 b, only the $42 \mathrm{~ms}$ condition was tested, and maintaining fixation during stimulus presentation was defined as keeping gaze within a $4.4^{\circ} \times 4.4^{\circ}$ square surrounding the initial fixation location until the test picture's onset.

\section{Results and Discussion}

Again, observers tended to be rather confident of their responses. On average, the percentages of "sure," "pretty sure," and "not sure" responses were 26\%, 56\%, and 16\%, respectively. Observers failed to recognize pictures on only $2 \%$ of the trials, and these were excluded from all analyses. Separate ANOVAs conducted on observers' mean boundary ratings on maintainfixation and shift-gaze trials revealed no main effect of side of stimulus presentation and no interaction with serial position in either condition in either experiment (all $F \mathrm{~s}<1.66, n$.s.). 
Mean boundary ratings in each of these conditions were subsequently collapsed across the factor of side of stimulus presentation in both experiments.

Experiment 3a: Serial Position 3-Again, the critical condition for observing time course is when the target is in the final position in the sequence. Replicating Experiment 2, when fixation was maintained, BE occurred for the final picture at each masked interval. The mean boundary ratings and 95\% confidence intervals are shown in Figure 6 (left panel). This provides additional evidence to suggest that BE occurred rapidly enough following stimulus offset to be present in transsaccadic memory. BE for the final picture in the sequence also survived a gaze shift for all masked intervals tested. The mean boundary ratings and $95 \%$ confidence intervals for shift-gaze trials are shown in Figure 6 (right panel). This shows that BE survived a gaze shift early in processing.

In the maintain-fixation condition, planned comparisons among all three masked intervals showed that when fixation was maintained, the mean rating for serial position 3 targets at the 42-ms masked interval was significantly smaller than both the mean rating for serial position 3 targets at the $100-\mathrm{ms}$ interval $(-.08$ vs. -.25$), t(70)=2.73, p<.05$, and the mean rating for those at the 250 -ms interval $(-.08$ vs. -.36$), t(70)=4.22, p<.05$.

Experiment 3a: Serial Positions 1 and 2-As in Experiments 1 and 2, BE was not eliminated by conceptual masking. As can be seen from the $95 \%$ confidence intervals for maintain-fixation trials (Figure 6, left panel), and shift-gaze trials (Figure 6, right panel), targets that appeared in serial positions 1 and 2 yielded significant BE. In the context of visual scanning, this is important because the contents of each new fixation would serve as a conceptual mask for the contents of the previous one.

Experiment 3b: Oculomotor Data-BE occurred for targets in all three serial positions in both conditions, as is shown in Figure 7. The eye tracking data revealed that observers maintained fixation during stimulus presentation on $84 \%$ of maintain-fixation trials and $79 \%$ of shift-gaze trials. An ANOVA comparing observers' mean boundary ratings for all trials to their mean ratings for only trials on which fixation was maintained revealed no changes in data patterns (all $F \mathrm{~s}<1$ ). Thus observers were following instructions, rather than adopting a strategy of fixating near an edge instead of at the center of the stimuli.

The eye tracking data also revealed that the average retention interval on shift-gaze trials, defined as the time between the offset of the final scene in the RSVP sequence and the beginning of the first fixation on the test picture, was $370 \mathrm{~ms}$. This interval can be divided into three components: the masked interval (during which fixation was maintained; $42 \mathrm{~ms}$ ) the saccade latency (the time required to initiate the saccade following test picture onset; $264 \mathrm{~ms}$ ), and the saccade duration (64 ms). Leftward and rightward saccade latencies did not differ significantly [255 ms vs. $273 \mathrm{~ms} ; t(35)=1.29$, n.s.], nor did the corresponding retention intervals for leftside or right-side test items $[360 \mathrm{~ms}$ vs. $381 \mathrm{~ms} ; t(35)=1.44, n . s$. ]. Thus the time required for observers to shift gaze to the test picture on shift-gaze trials was only slightly longer than typical saccade latencies, which are considered to be in the area of 150 to $200 \mathrm{~ms}$ (Carpenter, 1988; Rayner, 1998). This suggests that observers were neither delaying saccades to test pictures nor initiating them prior to the test picture's onset.

In conclusion, the results of Experiments $3 \mathrm{a}$ and $3 \mathrm{~b}$ show that BE would, at the least, be available to play a role in the integration of successive views and that it is clearly robust enough to survive a shift in gaze following a dynamic series of briefly presented scenes. 


\section{General Discussion}

Boundary extension is an error of commission. One usually thinks of such errors as emerging over relatively long periods of time or under conditions of high memory load. In the present experiments we demonstrated that an interruption of a view for as little as $42 \mathrm{~ms}$ was sufficient to elicit boundary extension. In these cases observers misidentified the identical view as looking more close up than the view seen before the interruption. Across experiments, we tested memory for target pictures embedded in rapidly presented streams of three pictures followed by a masked interval of $1 \mathrm{~s}, 625 \mathrm{~ms}, 250 \mathrm{~ms}, 100 \mathrm{~ms}$, or $42 \mathrm{~ms}$. Boundary extension occurred following all of these intervals and did so irrespective of the target's serial position.

At the outset we had expected that an early visual memory buffer, perhaps iconic memory (which can sometimes persist in spite of a mask; Smithson \& Mollon, 2006) or VSTM, might prevent the involvement of boundary extension in the earliest phases of visual scanning, but clearly, at least in the presence of a disruptive mask, boundary extension was evident following an interval commensurate with a saccade. In addition, in all three experiments, BE occurred for pictures that appeared in serial positions 1 and 2, suggesting that conceptual masking - the immediate onset of new, meaningful visual information - does not eliminate boundary extension (Intraub, 1984, 1999; Potter, 1976, 1999).

Experiments 1, 3a, and $3 \mathrm{~b}$ demonstrated that this early, potentially fragile memory of an extended view was robust enough to survive a shift in gaze. This is important because it shows that neither the attention shift nor motor planning involved in this act disrupted what was, for the most part, a rather confidently held assessment that the original view had been more expansive. This occurred both when observers knew exactly where the test picture would appear (Experiments 3a and 3b) and when they had no advance warning and the test picture's spatial position shifted randomly across trials (Experiment 1). These experiments exploited one of the most striking characteristics of BE-the failure to recognize an identical close-up view.

Experiment 2 provided converging evidence for the occurrence of boundary extension by testing for additional patterns of results that are diagnostic of boundary extension under conditions in which observers maintained fixation. Observers were presented with close-up or more wide-angle views and test pictures were either identical to the target or showed the alternate view (e.g., Gottesman \& Intraub, 2002; Intraub et al., 1992, 1998; Intraub \& Richardson, 1989). As in the other experiments, repetition of the same close-up at presentation and test was not recognized, as observers tended to think the repetition was more "close-up." However, it also showed that repetition of the same wider angle views resulted in less boundary extension than had been obtained for the repeated close-ups and that a robust asymmetry occurred for trials on which a different view was shown at test. These patterns occurred not only for the last position in the sequence, but across all serial positions, again demonstrating that BE was not eliminating by conceptual masking (Intraub, 1984, 1999; Potter, 1976, 1999).

To test the early time course of BE it was necessary to follow a target picture with the test picture, so of course our test conditions did not provide a simulation of what usually happens during visual scanning in which the image changes with each saccade. It is important to note, however, that our test provides an even stronger test of BE for that very reason. Given that BE occurred both when the same picture repeated and when the observer could control an eye movement with full knowledge that spatial memory would be tested, it is reasonable to conclude that $\mathrm{BE}$ is, at the very least, available early enough in processing to play a role in view integration during active visual scanning. 


\section{Implications for Transsaccadic Memory and Scene Representation}

Most researchers agree that scene representation is not picture-like, but instead is more schematic in nature (e.g., Henderson \& Hollingworth, 2003; Hochberg, 1978, Intraub, 1997, Simons \& Levin, 1997; Simons \& Rensink, 2005). The schematic character of representation has also been posited specifically in the case of transsaccadic memory (Carlson-Radvansky, 1999; Carlson-Radvansky \& Irwin, 1995; Irwin, 1991, 1992; Verfaillie \& De Graef, 2000;

Verfaillie et al., 1994). Research has demonstrated that scene layout is obtained rapidly and that in addition to conveying a rough portrait of scene structure, it supports scene comprehension at a conceptual level (Biederman, 1981; Oliva \& Schyns, 1997). The present research demonstrates that not only is some characterization of layout maintained in transsaccadic memory, but that this representation includes a projection of the layout beyond the bounds of the original sensory information. The rapidity of this effect is striking.

Although it is assumed that some representation of layout is retained above and beyond the individual details of a view (e.g., Rensink, 2000), the current experiments provide evidence that it is retained within transsaccadic memory as well. This adds to our knowledge of the transsaccadic representation because most research in that area has focused primarily on important issues of object representation. For example, several studies have addressed the number of objects (Irwin, 1991, 1992; Irwin \& Andrews, 1996) and the types of object properties (Gordon \& Irwin, 1996; Henderson, 1997; Henderson \& Siefert, 1999, 2001) that can be maintained across a saccade. There has also been research on spatial relations that are maintained across a saccade, both among parts of the same object (Carlson-Radvansky \& Irwin, 1995; Verfaillie \& De Graef, 2000) and between multiple objects (Carlson-Radvansky, 1999; Deubel, 2004; Germeys et al., 2004). The current research suggests, somewhat surprisingly, that a visual interruption lasting the duration of a saccade is enough time for boundary extension to occur.

The extended region is very constrained, perhaps enough to prime upcoming layout (Sanocki, 2003), but not so much that we confabulate and become confused during visual scanning. This unidirectional boundary error might better be classified as a highly constrained prediction rather than an error. After all, the goal of the visual system is to construct a coherent representation of the surrounding visual world, in spite of the fact that scene perception is built from a succession of samples over time. Successive fixations embellish the representation with more detailed information (Hollingworth, 2004; Hollingworth \& Henderson, 2002; Melcher, 2001, 2006; Zelinsky \& Loschky, 2005). But the location of view-boundaries is not among those details. It is more adaptive for the visual system to "ignore" these spurious boundaries and extrapolate the likely continuity of global layout just beyond them.

\section{Implications for the Locus of Boundary Extension}

Having more clearly charted boundary extension's early time course, we return to our original question: At what stage of processing of a given view does BE occur-perception or memory? Finding evidence for BE in memory following a 42-ms gap in sensory input suggests that BE occurs within the span of a single saccade - perhaps as soon as sensory input is removed. What implications do these findings have for the locus of boundary extension? Is there a "process" of extrapolation that rapidly takes place in memory during the brief gap between fixations? A more likely possibility is that the first step of the creation of a boundary-extended view involves processes taking place during sensory perception (i.e., while the sensory information is present). Here it is important to entertain the role of amodal perception and higher order expectation in scene perception. The layout and gist of a scene are grasped very rapidlyrequiring only a brief glimpse (less than the duration of a typical fixation; Biederman, 1981; Intraub, 1981; Oliva \& Schyns, 1997; Potter, 1976; Thorpe, Fize, \& Marlot, 1996). Amodal perception of surfaces (Kellman, Yin, \& Shipley, 1998; Yin, Kellman, \& Shipley, 1997, 
2000) and objects (Kanizsa, 1979) that are cropped by the edges of the view would be expected to occur simultaneously. Thus, while the stimulus is visible, the observer's representation includes both the sensory information contained in the view and the unseen layout beyond its edges.

While the stimulus is in view, the observer can readily note where the periphery of the picture terminates and the amodally generated part of the representation begins. Once the sensory input is gone, however, the distinction between what was sensory and what was amodal is no longer clear. The representation of the highly expected, amodally generated region just beyond the view may be mistaken as having been seen, thus resulting in boundary extension. In a sense then, the boundary-extended region is already a part of the representation before the stimulus is gone (albeit an amodal part). Only without sensory input is it mistaken for having been seen. Thus, we observed boundary extension in Experiments 2, 3a, and 3b under extremely artificial conditions that would be expected to support a far better memory representation than what would be expected under normal visual scanning. When fixation was maintained, BE occurred following a $42 \mathrm{~ms}$ break in the sensory input when the identical view appeared in the same spatial location.

In conclusion, this series of experiment demonstrates the likelihood that boundary extension is available in transsaccadic memory early enough to help place each successive view in its larger spatial framework (Intraub, 2002; Intraub et al., 1992) and to play a role in the integration of successive views. It is well accepted that transsaccadic memory does not retain a point-bypoint representation of the world. The current research shows that the transsaccadic representation of expanse is not simply a vague representation that fluctuates with random error. Instead, the spatial representation includes a constructive unidirectional error that would serve as a good prediction of the continuity of layout. We suggest that this rapid projection of layout may be one of the means by which the visual system supports perception of a continuous, coherent world that can never be seen all at once but must be sampled a part at a time.

\section{Acknowledgments}

This research was supported by NIMH Grant RO1MH54688. We thank Daniel Bensonoff, Kari Schweifel, Jason Rand, Mattie Wilson, and Dave Drowos for their valuable assistance in preparing stimuli and collecting data.

\section{References}

Bertamini M, Jones LA, Spooner A, Hecht H. Boundary extension: The role of magnification, object size, context, and binocular information. Journal of Experimental Psychology: Human Perception and Performance 2005;31(6):1288-1307. [PubMed: 16366790]

Biederman, I. On the semantics of a glance at a scene.. In: Kubovy, M.; Pomerantz, JR., editors. Perceptual organization. Erlbaum; Hillsdale, NJ: 1981. p. 213-253.

Candel I, Merckelbach H, Zandbergen M. Boundary distortions for neutral and emotional pictures. Psychonomic Bulletin \& Review 2003;10(3):691-695. [PubMed: 14620365]

Carlson-Radvansky LA. Memory for relational information across eye movements. Perception \& Psychophysics 1999;61(5):919-934. [PubMed: 10499004]

Carlson-Radvansky LA, Irwin DE. Memory for structural information across eye movements. Journal of Experimental Psychology: Learning, Memory, and Cognition 1995;21(6):1441-1458.

Carpenter, RHS. Movements of the Eyes. Pion; London: 1988.

Deubel H. Localization of targets across saccades: Role of landmark objects. Visual Cognition 2004;11 (23):173-202.

Deubel H, Schneider WX. Saccade target selection and object recognition: Evidence for a common attentional mechanism. Vision Research 1996;36(12):1827-1837. [PubMed: 8759451]

Epstein R, Kanwisher N. A cortical representation of the local visual environment. Nature 1998;392(9): 598-601. [PubMed: 9560155] 
Germeys F, de Graef P, Panis S, van Eccelpoel C, Verfaillie K. Transsaccadic integration of bystander locations. Visual Cognition 2004;11(23):203-234.

Gordon RD, Irwin DE. What's in an object file? Evidence from priming studies. Perception \& Psychophysics 1996;58(8):1260-1277. [PubMed: 8961836]

Gottesman CV, Intraub H. Surface construal and the mental representation of scenes. Journal of Experimental Psychology: Human Perception and Performance 2002;28(3):589-599. [PubMed: 12075890]

Gottesman CV, Intraub H. Constraints on spatial extrapolation in the mental representation of scenes: View-boundaries vs. object-boundaries. Visual Cognition 2003;10(7):875-893.

Henderson JM. Two representational systems in dynamic visual identification. Journal of Experimental Psychology: General 1994;123(4):410-426. [PubMed: 7996123]

Henderson JM. Transsaccadic memory and integration during real-world object perception. Psychological Science 1997;8(1):51-55.

Henderson JM, Hollingworth A. Global transsaccadic change blindness during scene perception. Psychological Science 2003;14:493-497. [PubMed: 12930482]

Henderson JM, Siefert ABC. The influence of enantiomorphic transformation on transsaccadic object integration. Journal of Experimental Psychology: Human Perception and Performance 1999;25(1): 243-255.

Henderson JM, Siefert ABC. Types and tokens in transsaccadic object identification: Effects of spatial position and left-right orientation. Psychonomic Bulletin \& Review 2001;8(4):753-760. [PubMed: 11848596]

Hochberg, J. Perception. Vol. 2nd ed.. Prentice-Hall; Englewood Cliffs, NJ: 1978.

Hoffman JE, Subramaniam B. The role of visual attention in saccadic eye movements. Perception and Psychophysics 1995;57(6):787-795. [PubMed: 7651803]

Hollingworth A. Constructing visual representations of natural scenes: The roles of short- and long-term visual memory. Journal of Experimental Psychology: Human Perception and Performance 2004;30 (3):519-537. [PubMed: 15161384]

Hollingworth A, Henderson JM. Accurate visual memory for previously attended objects in natural scenes. Journal of Experimental Psychology: Human Perception and Performance 2002;28(1):113136.

Hollingworth A, Hyun J-S, Zhang W. The role of visual short-term memory in empty cell localization. Perception \& Psychophysics 2005;67(8):1332-1343. [PubMed: 16555585]

Intraub H. Rapid conceptual identification of sequentially presented pictures. Journal of Experimental Psychology: Human Perception and Performance 1981;7(3):604-610.

Intraub H. Conceptual masking: The effects of subsequent visual events on memory for pictures. Journal of Experimental Psychology: Learning, Memory, and Cognition 1984;10(1):115-125.

Intraub H. The representation of visual scenes. Trends in Cognitive Sciences 1997;1(6):217-221.

Intraub, H. Understanding and remembering briefly glimpsed pictures: Implications for visual scanning and memory.. In: Coltheart, V., editor. Fleeting memories: Cognition of brief visual stimuli. MIT Press; Cambridge, MA: 1999. p. 47-70.

Intraub H. Anticipatory spatial representation of natural scenes: Momentum without movement? Visual Cognition 2002;9(12):93-119.

Intraub, H. Scene perception: The world through a window.. In: Peterson, MA.; Gillam, B.; Sedgwick, HA., editors. Mental Structure in Visual Perception: Julian Hochberg's Contributions to Our Understanding of the Perception of Pictures, Film, and the World. Oxford University Press; New York: 2007. p. 454-466.

Intraub H, Bender RS, Mangels JA. Looking at pictures but remembering scenes. Journal of Experimental Psychology: Learning, Memory, and Cognition 1992;18(1):180-191.

Intraub H, Berkowits D. Beyond the edges of a picture. American Journal of Psychology 1996;109(4): 581-598.

Intraub, H.; Dickinson, CA. Boundary extension at a glance and across an eye movement.. Paper presented at the annual meeting of the Psychonomic Society; Houston, TX. Nov. 2006 
Intraub H, Gottesman CV, Bills AJ. Effects of perceiving and imagining scenes on memory for pictures. Journal of Experimental Psychology: Learning, Memory, and Cognition 1998;24(1):186-201.

Intraub H, Gottesman CV, Willey EV, Zuk IJ. Boundary extension for briefly glimpsed photographs: Do common perceptual processes result in unexpected memory distortions? Journal of Memory and Language 1996;35:118-134.

Intraub H, Hoffman JE, Wetherhold CJ, Stoehs S-A. More than meets the eye: The effect of planned fixations on scene representation. Perception \& Psychophysics 2006;68(5):759-769. [PubMed: 17076344]

Intraub H, Richardson M. Wide-angle memories of close-up scenes. Journal of Experimental Psychology: Learning, Memory, and Cognition 1989;15(2):179-187.

Irwin DE. Information integration across saccadic eye movements. Cognitive Psychology 1991;23:420456. [PubMed: 1884598]

Irwin DE. Memory for position and identity across eye movements. Journal of Experimental Psychology: Learning, Memory, and Cognition 1992;18(2):307-317.

Irwin, DE. Perceiving an integrated visual world.. In: Meyer, DE.; Kornblum, S., editors. Attention and Performance 14: Synergies in Experimental Psychology, Artificial Intelligence, and Cognitive Neuroscience. The MIT Press; Cambridge, MA: 1993. p. 121-142.

Irwin, DE.; Andrews, RV. Integration and accumulation of information across saccadic eye movements.. In: Inui, T.; McClelland, JL., editors. Attention and Performance XVI: Information Integration in Perception and Communication. MIT Press; Cambridge, MA: 1996. p. 125-155.

Irwin DE, Brockmole JB. Suppressing where but not what: The effect of saccades on dorsal- and ventralstream visual processing. Psychological Science 2004;15(7):467-473. [PubMed: 15200631]

Irwin DE, Brown JS. Tests of a model of informational persistence. Canadian Journal of Psychology 1987;41(3):317-338. [PubMed: 3502903]

Irwin DE, Yeomans JM. Sensory registration and informational persistence. Journal of Experimental Psychology: Human Perception and Performance 1986;12(3):343-360. [PubMed: 2943863]

Irwin DE, Zelinsky G. Eye movements and scene perception: Memory for things observed. Perception \& Psychophysics 2002;64:882-895. [PubMed: 12269296]

Kanizsa, G. Organization in Vision. Praeger; New York: 1979.

Kellman PJ, Yin C, Shipley TF. A common mechanism for illusory and occluded object completion. Journal of Experimental Psychology: Human Perception and Performance 1998;24(3):859-869. [PubMed: 9627421]

Koriat A, Goldsmith M, Pansky A. Toward a psychology of memory accuracy. Annual Review of Psychology 2000;51:481-537.

Kowler E, Anderson E, Dosher B, Blaser E. The role of attention in the programming of saccades. Vision Research 1995;35(13):1897-1916. [PubMed: 7660596]

Mathews A, Mackintosh B. Take a closer look: Emotion modifies the boundary extension effect. Intelligence 2004;32(2):217-219.

Melcher D. Persistence of visual memory for scenes. Nature 2001;412(6845):401. [PubMed: 11473303]

Melcher D. Accumulation and persistence of memory for natural scenes. Journal of Vision 2006;6(1):817. [PubMed: 16489855]

Oliva A, Schyns PG. Coarse blobs or fine edges? Evidence that information diagnosticity changes the perception of complex visual stimuli. Cognitive Psychology 1997;34:72-107. [PubMed: 9325010]

Olson IR, Jiang Y. Visual short-term memory is not improved by training. Memory \& Cognition 2004;32 (8):1326-1332.

Park SJ, Intraub H, Yi D-J, Widders D, Chun MM. Beyond the edges of a view: Boundary extension in human scene-selective visual cortex. Neuron 2007;54(2):335-342. [PubMed: 17442252]

Phillips WA. On the distinction between sensory storage and short-term visual memory. Perception \& Psychophysics 1974;16(2):293-290.

Potter MC. Short-term conceptual memory for pictures. Journal of Experimental Psychology: Human Learning and Memory 1976;2(5):509-522. [PubMed: 1003124] 
Potter, MC. Understanding sentences and scenes: The role of conceptual short-term memory.. In: Coltheart, V., editor. Fleeting memories: Cognition of brief visual stimuli. MIT Press; Cambridge, MA: 1999. p. 13-46.

Previc FH, Intraub H. Vertical biases in scene memory. Neuropsychologica 1997;35(12):1513-1517.

Rayner K. Eye movements in reading and information processing: 20 years of research. Psychological Bulletin 1998;124(3):372-422. [PubMed: 9849112]

Rensink RA. The dynamic representation of scenes. Visual Cognition 2000;7(1-3):17-42.

Sanocki T. Representation and perception of scenic layout. Cognitive Psychology 2003;47:43-86. [PubMed: 12852935]

Simons DJ, Levin DT. Change blindness. Trends in Cognitive Science 1997;1:261-267.

Simons DJ, Rensink RA. Change blindness: Past, present, and future. Trends in Cognitive Sciences 2005;9(1):16-20. [PubMed: 15639436]

Smithson H, Mollon J. Do masks terminate the icon? The Quarterly Journal of Experimental Psychology 2006;59(1):150-160. [PubMed: 16556564]

Thorpe S, Fize D, Marlot C. Speed of processing in the human visual system. Nature 1996;381(6):520522. [PubMed: 8632824]

Verfaillie K, De Graef P. Transsaccadic memory for position and orientation of saccade source and target. Journal of Experimental Psychology: Human Perception and Performance 2000;26(4):1243-1259. [PubMed: 10946713]

Verfaillie K, De Troy A, Van Rensbergen J. Transsaccadic integration of biological motion. Journal of Experimental Psychology: Learning, Memory, and Cognition 1994;20:649-670.

Volkmann FC. Human visual suppression. Vision Research 1986;26(9):1401-1416. [PubMed: 3303665]

Yin C, Kellman PJ, Shipley TF. Surface completion complements boundary interpolation in the visual integration of partly occluded objects. Perception 1997;26(11):1459-1479. [PubMed: 9616474]

Yin C, Kellman PJ, Shipley TF. Surface integration influences depth discrimination. Vision Research 2000;40(15):1969-1978. [PubMed: 10828465]

Zelinsky GJ, Loschky LC. Eye movements serialize memory for objects in scenes. Perception \& Psychophysics 2005;67:676-690. [PubMed: 16134461] 


\section{Close-Up View}

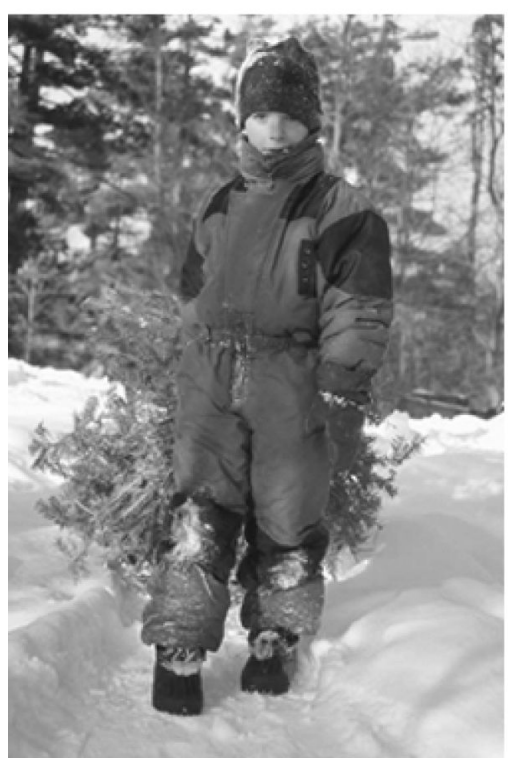

\section{Wide-Angle View}

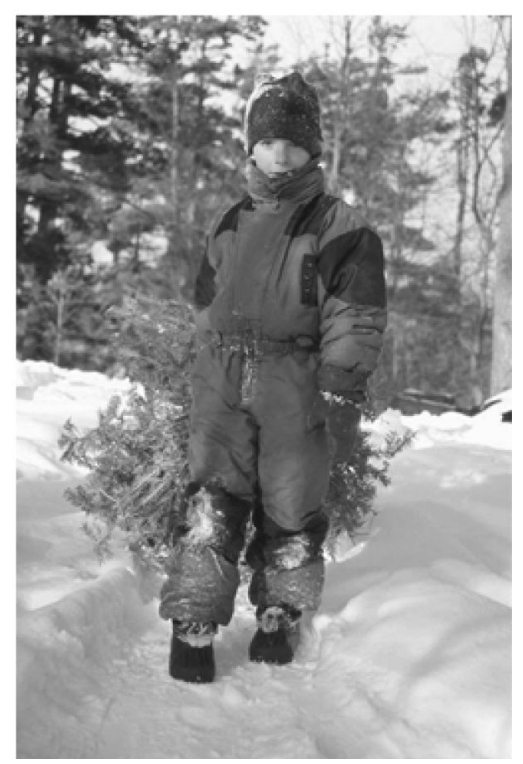

Figure 1.

A close-up version of a target picture is shown on the left, and wider angle version of the same target picture is shown on the right. 


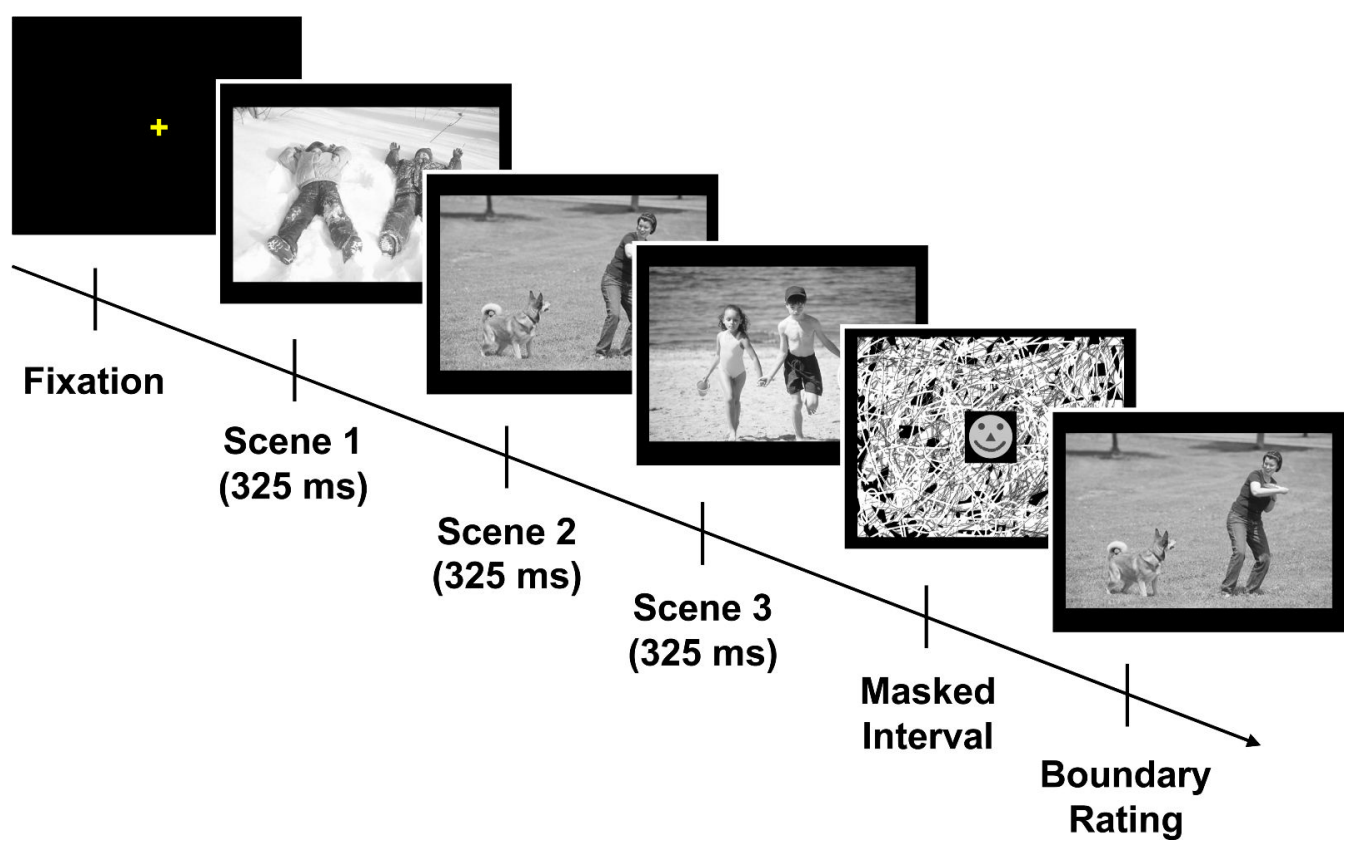

Figure 2.

A schematic illustration of a trial sequence with the test picture in the center of the screen (maintain-fixation condition). Note that the actual stimuli did not fill the screen, as is shown in the example. 
Serial Position

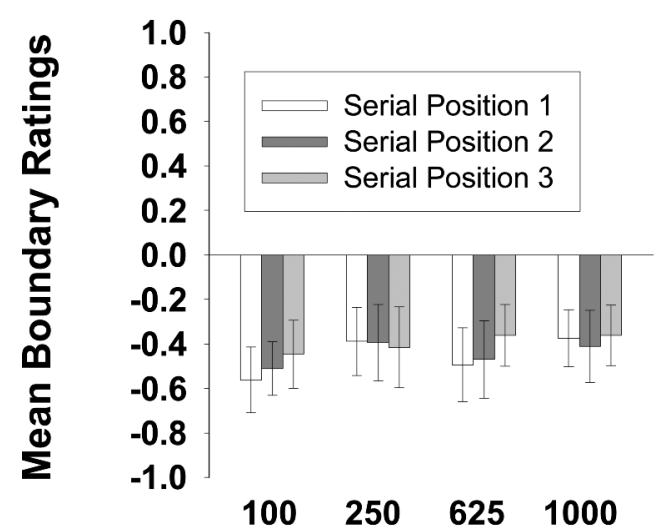

$\begin{array}{llll}100 \quad 250 & 625 & 1000\end{array}$
Spatial Position

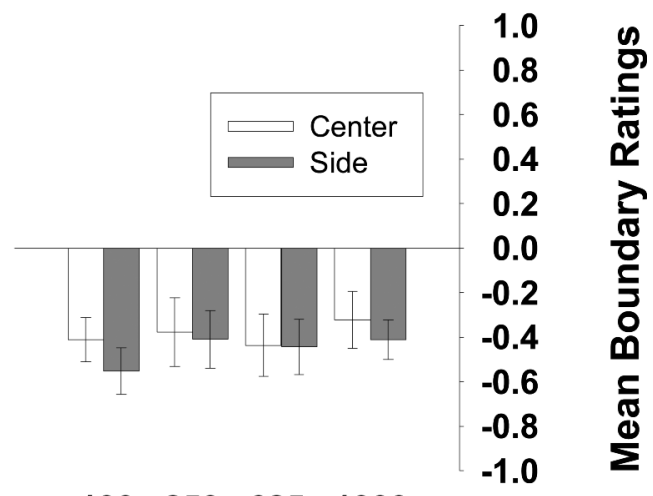

1002506251000

\section{Masked Interval (ms)}

Figure 3.

Observers' mean boundary ratings for each serial position of the target (collapsed across spatial position of the test picture) are shown on the left, and their mean boundary ratings for each spatial position of the test picture (center, side; collapsed across serial position of the target) are shown on the right, for each retention interval (Experiment 1). All error bars show the $95 \%$ confidence interval of the mean. Means that are significantly less than zero reflect boundary extension; means that are significantly greater than zero reflect boundary restriction. 
Close-Wide

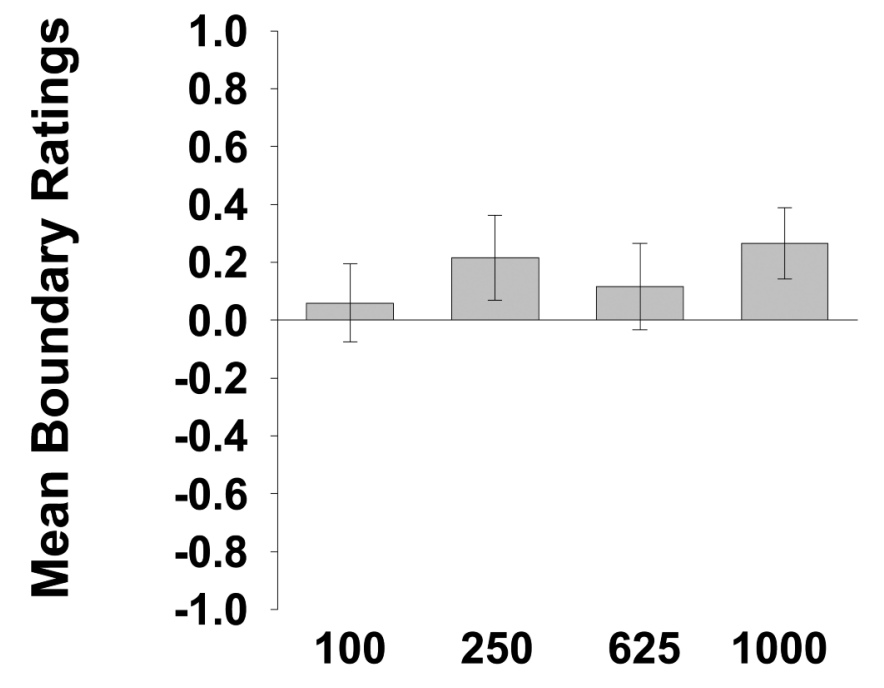

\section{Wide-Close}

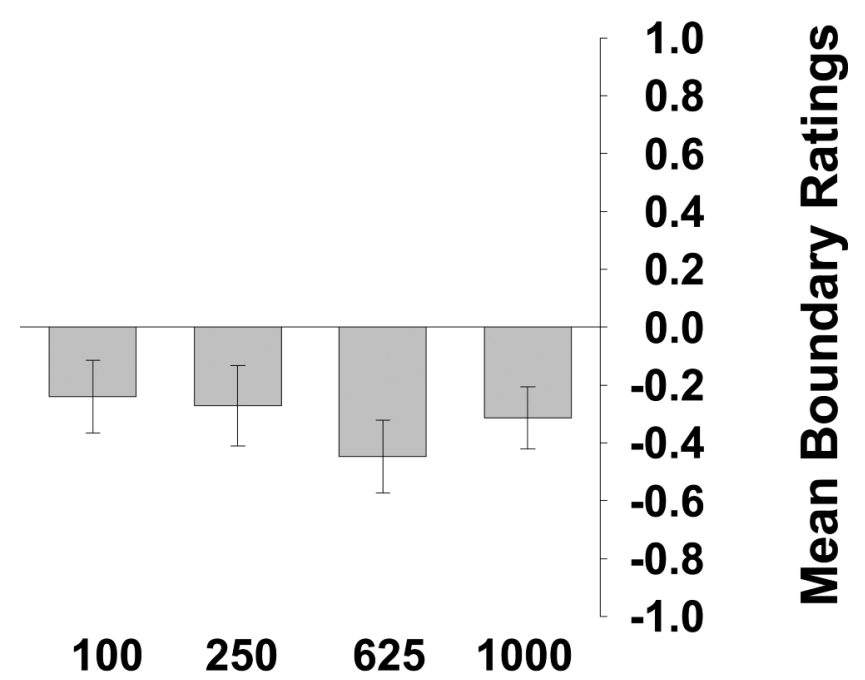

\section{Masked Interval (ms)}

Figure 4.

Observers' mean boundary ratings (collapsed across both serial position of the target and spatial position of the test picture) for trials on which a close-up target was tested by wide-angle view are shown on the left, and their mean boundary ratings for trials on which a wide-angle target was tested by close-up view are shown on the right, for each retention interval (Experiment 1). 
Close-Close

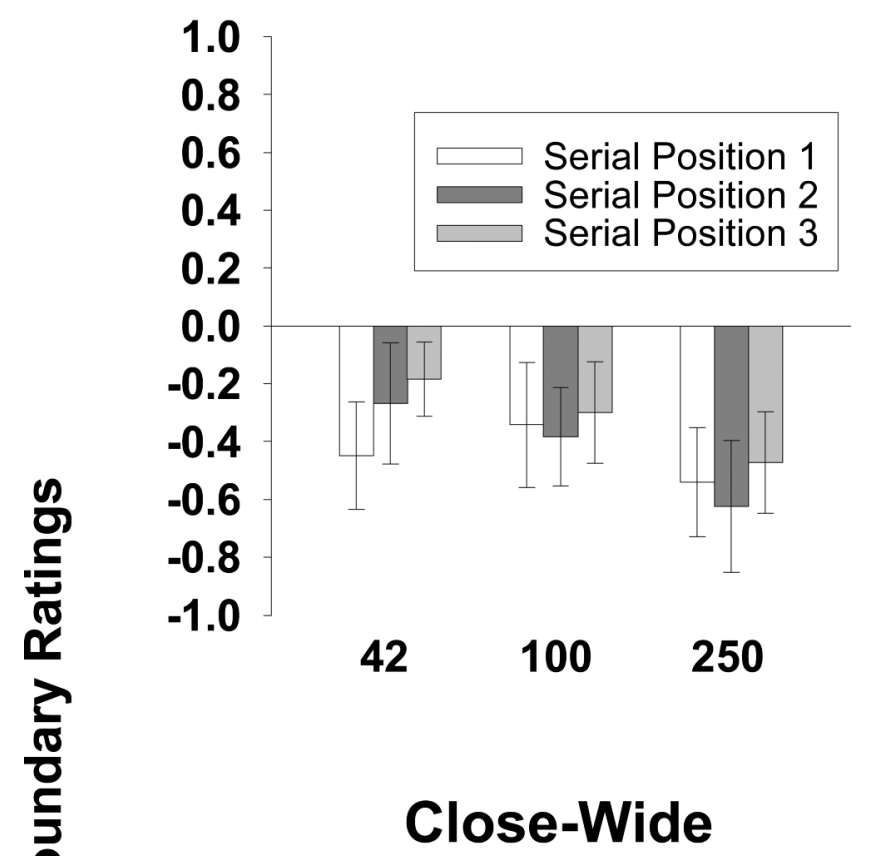

Wide-Wide

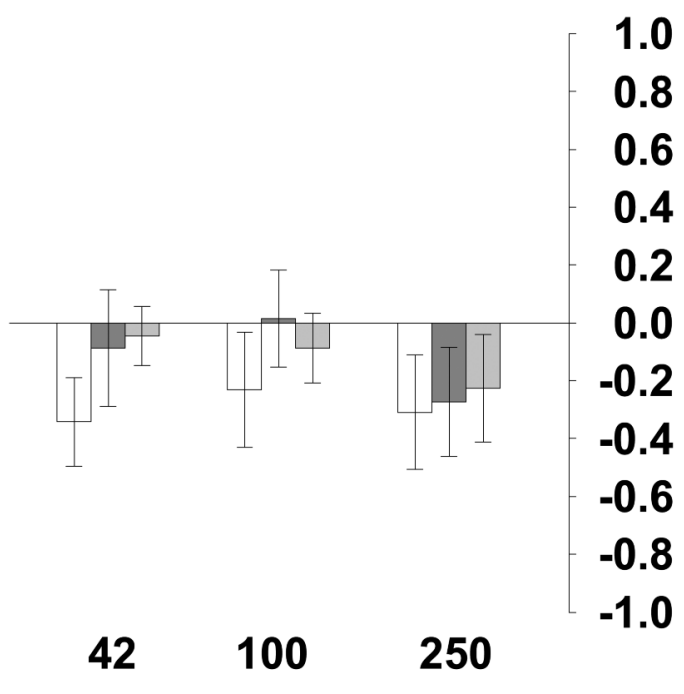

Wide-Close

1.0
0.8
0.6
0.4
0.2
0.0
-0.2
-0.4
-0.6
-0.8
-1.0

42

100

250

\section{Masked Interval (ms)}

Figure 5.

Observers' mean boundary ratings for CC trials are shown on the upper left, their mean boundary ratings for WW trials are shown on the upper right, their mean boundary ratings for CW trials are shown on the lower left, and their mean boundary ratings for WC trials are shown on the lower right, for each serial position of the target at each retention interval (Experiment 2). 
Maintain Fixation

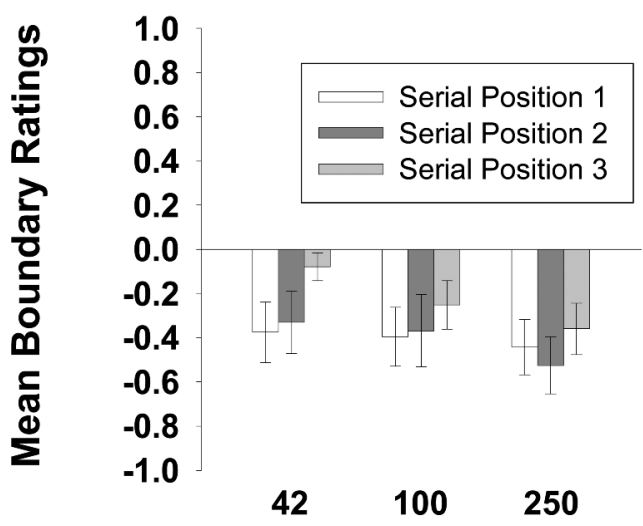

Shift Gaze

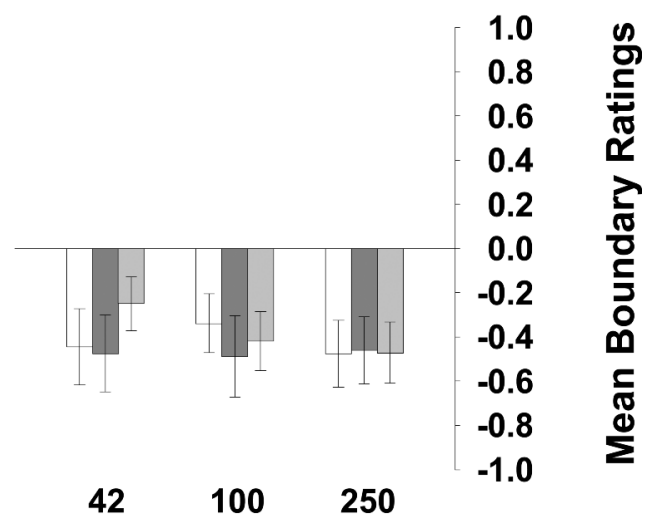

\section{Masked Interval (ms)}

Figure 6.

Observers' mean boundary ratings for maintain-fixation trials are shown on the left, and their mean boundary ratings for shift-gaze trials are shown on the right, for each serial position of the target at each retention interval (Experiment 3a). 


\section{Maintain Fixation}

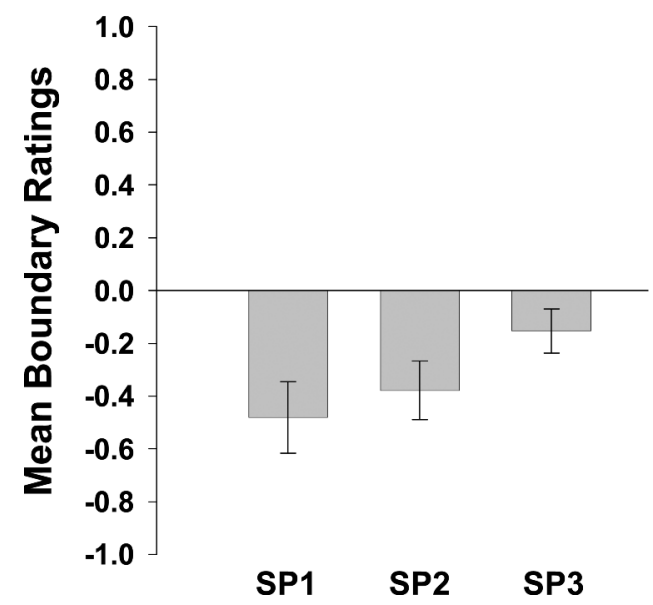

Shift Gaze

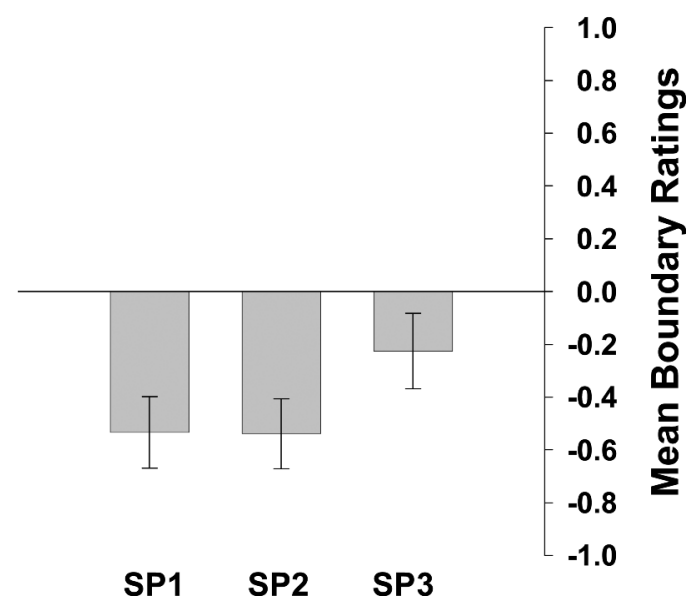

Figure 7.

Observers' mean boundary ratings for maintain-fixation trials are shown on the left, and their mean boundary ratings for shift-gaze trials are shown on the right, for each serial position (SP) of the target (Experiment 3b). 